\title{
O USO DE ESTRATÉGIAS MEDIADORAS NA PRÁTICA DE REVISÃO DE PRODUÇÃO ESCRITA POR PARES
}

\section{THE USE OF MEDIATING STRATEGIES DURING PEER REVIEW IN WRITING}

\author{
Daniele Blos Bolzan ${ }^{1}$
}

\begin{abstract}
RESUMO: Pesquisas têm indicado que o diálogo colaborativo poderia ser o lugar onde o uso da língua e a aprendizagem da língua poderiam coocorrer (SWAIN, 1997). Este artigo irá apresentar um recorte de uma pesquisa (BLOS BOLZAN, 2016) que teve por objetivo propor a prática de revisão por pares de produção escrita a um $8^{o}$ ano do Ensino Fundamental de uma escola com currículo bilíngue Português-Inglês do sul do Brasil. Esse recorte, por sua vez, teve por objetivo exemplificar quais estratégias mediadoras foram utilizadas pelos participantes da prática de revisão por pares e analisar como elas facilitaram o cumprimento da tarefa - no caso, a correção dos textos. Pelos exemplos apresentados neste artigo, foi possível perceber que os aprendizes utilizaram uma variedade de estratégias mediadoras, unindo seus conhecimentos, ao trabalharem juntos alcançando níveis mais elevados de desempenho através da assistência mútua.
\end{abstract}

PALAVRAS-CHAVE: Estratégias Mediadoras; Revisão Por Pares; Colaboração; Produção Escrita.

ABSTRACT: Research has shown that collaborative dialogue could be where language use and language learning would co-occur (SWAIN, 1997). This article will present partial data from a broader research (BLOS BOLZAN, 2016) whose objective was the implementation of peer review in writing in an $8^{\text {th }}$ grade elementary class from a Portuguese-English bilingual school in the South of Brazil. The objective of the data presented in this article is to exemplify which mediating strategies were used by the participants of the peer review practice and analyze how they facilitated the accomplishment of the task - the text correction. It was possible to notice that learners used a variety of mediating strategies, gathering their knowledge when working together and reaching greater levels of performance through mutual assistance.

KEYWORDS: Mediating Strategies; Peer Review; Collaboration; Writing.

\section{Introdução}

\footnotetext{
${ }^{1}$ Doutora em Linguística Aplicada pela UFRGS. Coordenadora da área de Línguas Estrangeiras e professora de Língua Inglesa da Instituição Evangélica de Novo Hamburgo.
} 
Os dados apresentados neste artigo são um recorte de uma pesquisa realizada em contexto de educação bilíngue (BLOS BOLZAN, 2016). A proposta de ensino bilíngue da escola participante tem como objetivo acrescentar uma outra língua majoritária ao repertório do aprendiz (que muitas vezes só é monolíngue em português), configurando, assim, no que se denomina educação bilíngue de escolha (CAVALCANTI, 1999) ou de enriquecimento (HORNBERGER, 1991).

Em relação a essa modalidade de educação bilíngue, pesquisas no contexto canadense vêm observando ao longo dos tempos que os estudantes com formação em escolas bilíngues tornam-se rapidamente capazes de entender e ser entendidos na segunda língua (L2) e que a partir do momento em que a comunicação em L2 torna-se fluente, o desenvolvimento da L2 desacelera (SWAIN; LAPKIN, 1995), apesar da oportunidade que teriam de estar aprendendo a língua em um contexto significativo de comunicação (SATO, 2013). A sala de aula é rica de insumo e orientada para a comunicação, porém nem sempre há a necessidade do desenvolvimento contínuo da proficiência na L2, uma vez que o aluno consegue se comunicar através dela, apropriando-se dos conteúdos abordados (SWAIN, 1985). Nos programas de imersão, os alunos desenvolvem altos níveis de competência estratégica para lidar com os conteúdos e, como resultado, o crescimento da língua do aprendiz desacelera se não forem feitas tentativas de voltar a atenção do aluno para o modo como expressam os sentidos pretendidos (KOWAL; SWAIN, 1994). Assim, as pesquisas devem buscar alternativas de como ajudar os aprendizes inseridos em contexto de imersão (ou de educação bilíngue) a não só serem capazes de se apropriar dos conteúdos através da L2, mas também de continuar avançando de um estágio para outro na sua aprendizagem de L2. Penso ser esse também um desafio da escola bilíngue brasileira, que deve buscar formas de privilegiar, dentre seus objetivos, não só a fluência necessária para que o aluno consiga se apropriar dos conteúdos através da L2, mas que continue desenvolvendo a L2 em direção a formas mais acadêmicas de seu uso, na medida em que a complexidade curricular aumente.

O que as pesquisas têm indicado como uma possibilidade nesse sentido é a ênfase na interação e o uso da língua, tanto como uma ferramenta de mediação na realização de tarefas quanto como objeto de atenção para o foco na forma. Dessa forma, o diálogo colaborativo poderia ser o lugar onde o uso da língua e a aprendizagem da língua poderiam co-ocorrer (SWAIN, 1997, p. 117), já que seria a língua mediando a aprendizagem de língua, tornandose uma atividade cognitiva e social (SWAIN, 2000). Ao criar uma necessidade de comunicação para a realização de uma tarefa de forma colaborativa, os aprendizes são 
forçados a pensar sobre a forma de seu output linguístico e, assim, tornam-se conscientes das falhas de seu conhecimento linguístico (SWAIN; LAPKIN, 1995).

A partir dessa discussão, é possível afirmar, então, que uma forma de se avançar no contínuo desenvolvimento da proficiência dos aprendizes na L2 seria oferecendo mais oportunidade de participação do aprendiz em interações. Nesse sentido, a prática de revisão por pares viria ao encontro não só da necessidade dos alunos de desenvolverem a habilidade escrita, mas também de avançar no seu processo de aprendizagem de L2, atentando para a forma através das oportunidades de interação oferecidas.

\section{Metodologia}

Este estudo propôs a prática de revisão por pares de produção escrita a uma turma de $8^{\circ}$ ano do Ensino Fundamental de uma escola com currículo bilíngue português-inglês situada na região metropolitana de Porto Alegre, Rio Grande do Sul. Aliada à proposta de revisão por pares como forma de potencializar as aulas de produção escrita dentro de um módulo do currículo que prepara os alunos para a prova de Cambridge $F C E$, buscou-se promover a colaboração entre os participantes do evento de aprendizagem, acreditando que, através da interação, os aprendizes de L2, alunos da escola bilíngue, teriam a chance de atentar para as formas linguísticas, desenvolvendo, assim, sua proficiência linguística.

A turma participante era composta de 25 alunos de idades entre 12-14 anos e, para a análise, do total de duplas que realizaram as tarefas de produção textual e de revisão por pares, apenas quatro foram selecionadas para esta análise. Como requisito para esse recorte, buscou-se selecionar aquelas duplas que representassem diferentes perfis em relação a níveis de proficiência. Ou seja, em três duplas os participantes apresentavam nível de proficiência semelhante um ao outro (alto, baixo ou médio, em relação à média da turma da qual fazem parte) e uma dupla foi composta por participantes com nível de proficiência diferente (misto), sendo um aluno mais experiente em relação à língua inglesa do que o outro.

\begin{tabular}{|l|l|l|l|l|}
\hline Dupla & \multicolumn{1}{|c|}{ Nome $^{2}$} & $\begin{array}{c}\text { Idade quando } \\
\text { respondeu o } \\
\text { questionário }\end{array}$ & $\begin{array}{c}\text { Série em que } \\
\text { iniciou no } \\
\text { currículo } \\
\text { bilíngue }\end{array}$ & $\begin{array}{c}\text { Nível de } \\
\text { proficiência } \\
\text { (comparado à } \\
\text { média da turma) }\end{array}$ \\
\hline 1 & Ângelo & 14 & Nível 5 & Alto \\
\cline { 2 - 6 } & Bruno & 14 & Nível 4 & Alto \\
\hline
\end{tabular}

\footnotetext{
${ }^{2}$ Todos os nomes de participantes são pseudônimos.

3 "Nível 4" da Educação Infantil refere-se a duas séries antes do $1^{\circ}$ ano do Ensino Fundamental; "Nível 5" refere-se ao último ano da Educação Infantil, antes do $1^{\circ}$ ano do Ensino Fundamental.
} 


\begin{tabular}{|l|l|l|l|l|}
\hline 2 & Diego & 13 & Nível 4 & Baixo \\
\cline { 2 - 5 } & Leonardo & 13 & $1^{\circ}$ ano & Baixo \\
\hline \multirow{3}{*}{3} & Pedro & 13 & Nível 4 & Alto \\
\cline { 2 - 5 } & Nathan & 13 & Nível 4 & Baixo \\
\hline \multirow{2}{*}{4} & Fátima & 13 & Nível 4 & Médio \\
\cline { 2 - 5 } & Jéssica & 12 & Nível 4 & Médio \\
\hline
\end{tabular}

Tabela 1

Anterior à implementação da prática de revisão por pares nas aulas, houve um período de preparação dos alunos, que responderam a um questionário visando a analisar suas expectativas, seguido de uma fase de sensibilização e outra fase de preparação para a prática. Passadas essas fases iniciais, em um período que foi de maio a setembro do ano letivo, os alunos escreveram, revisaram em pares e reescreveram cinco produções textuais cada. Cada prática de revisão por pares consistiu em: uma lição sobre o gênero textual, o momento da escrita do texto, a troca de textos entre membros da dupla e a revisão com comentários no texto e na lista de revisão, a interação da dupla comentando as sugestões dadas e recebidas (gravada em áudio e vídeo), a reescrita do texto e, por fim, a entrega das duas versões do texto para a professora-pesquisadora. Os alunos foram encorajados a interagir sobre os textos em língua inglesa, porém não foram impedidos de usar a língua portuguesa (L1) quando julgassem necessário.

Após a vivência das sessões de revisão, os oitos participantes analisados foram entrevistados individualmente, em entrevistas semiestruturadas, com o objetivo de fornecer informações sobre a avaliação que fizeram da experiência de revisão por pares.

As estratégias mediadoras utilizadas pelos participantes durante a prática de revisão por pares foram analisadas com base no modelo de Villamil e Guerrero (1996), classificadas da seguinte forma:

\section{a) Uso de fontes externas}

Essa categoria engloba exemplos em que os alunos utilizam alguma fonte externa, como o professor, o dicionário, o livro didático, a gramática, anotações no caderno, etc. com o intuito de buscar uma solução para o conteúdo do Episódio Relacionado à Língua (ERL) ${ }^{4}$.

\section{b) Uso da L1}

\footnotetext{
${ }^{4}$ Um Episódio Relacionado à Língua (ERL - do inglês Language Related Episodes (LRE), segundo SWAIN; LAPKIN, 1995) é definido como qualquer parte de um diálogo onde aprendizes falam sobre a língua que estão produzindo, questionam seu uso e se corrigem ou corrigem outros. Eles podem ser a consolidação do conhecimento linguístico do aprendiz, enquanto este aplica seu conhecimento existente a velhos ou novos contextos.
} 
Nessa categoria, encontram-se exemplos de trechos em que os alunos utilizam a L1 como ferramenta de mediação para o processo de revisão.

\section{c) Uso de scaffolding}

Nessa categorização, são listados exemplos de estratégias de andaimento, tais como pedir por sugestão, fornecer sugestão, responder à sugestão recebida, solicitar esclarecimentos, fornecer esclarecimentos, fornecer justificativas para sugestão, reafirmar algo mencionado pelo par como forma de checar suas intenções, fazer perguntas e responder a perguntas.

\section{d) Apoio do conhecimento metalinguístico}

Nessa categoria, os participantes fazem uso do conhecimento que têm da L2 e suas convenções para falar sobre suas sugestões.

\section{e) Uso do discurso privado}

Essa categoria engloba exemplos em que os alunos fazem comentários ou leem o texto para si mesmos, a fim de resolver um problema do texto sem a intenção de envolver o par.

\section{Análise}

Em uma atividade colaborativa como a prática de revisão por pares, os participantes utilizam-se de estratégias mediadoras que irão facilitar o cumprimento da tarefa - no caso, a correção dos textos -, cujo objetivo maior é o desenvolvimento da aprendizagem de L2 pelos participantes. Assim, a atividade mental do indivíduo será mediada e situada na interação social por meios simbólicos ou materiais. Os artefatos materiais irão mediar nossas ações através de objetos concretos, como um livro didático, um dicionário, o auxílio do professor ou de um colega, por exemplo; já os artefatos simbólicos irão mediar nossas ações através de representações simbólicas e abstratas, como o uso da L1 e L2, utilização de scaffolding, apoio no conhecimento metalinguístico e discurso privado (VYGOTSKY, 1978; LANTOLF, 2000; LANTOLF; THORNE, 2006). Cada um desses artefatos mediadores mencionados serão exemplificados e analisados a seguir, através de trechos selecionados e transcritos das interações dos alunos participantes ${ }^{5}$. Esses exemplos foram selecionados, dentre outros

\footnotetext{
5 ، " $\quad$ trecho do texto que está sendo lido.

$<\quad$ uso de português durante a interação.
} 
presentes nas interações, pois ilustram claramente o que se quer destacar em termos de estratégias mediadoras.

\section{O uso de fontes externas}

A estratégia mediadora utilizada pelos participantes da revisão por pares que fez uso de artefatos concretos foi o uso de fontes externas de consulta, tais como o livro didático, o dicionário, a lista de revisão, a professora e os colegas. Dentre esses, o uso do dicionário e a busca pelo auxílio da professora foram os observados com mais frequência. $\mathrm{O}$ dicionário era utilizado, principalmente, para conferência da ortografia de alguma palavra. Já a professora era consultada para diferentes tipos de auxílio, mas, em geral, acerca de questões que o dicionário não resolvia, tais como aspectos estruturais de frases e do texto.

Nos exemplos 1 e 2 é possível observar o uso do dicionário para a conferência de palavras. No exemplo 1, Ângelo confere a palavra "unfortunately" que havia sido escrita "unfortunetely" por Bruno em seu rascunho. No exemplo 2, Jéssica confere no dicionário a ortografia da palavra "theater", que Jéssica havia escrito "teather".

Exemplo 1 - Sessão de revisão por pares 3-10 de julho - Email - Dupla 1

Ângelo: And the spelling, is one time in "unfortunetely" (pega o dicionário para ver algo).

Bruno: <Acho que eu escrevi certo>.

Ângelo (checa o dicionário e compara com a forma escrita no texto, anota a forma certa no texto do colega): Un, un, for, for, tu, tu...

Bruno: Ah... "nately" (referindo-se à ortografia do final da palavra "unfortunately")

Exemplo 2 - Sessão de revisão por pares 3 - 10 de julho - Email - Dupla 4

Fátima: And "theater", I don't know if this is right. I just put... I just wrote.

Jéssica (pega o dicionário e olha): Like this (anota a forma correta). It's like this "theater".

Fátima: Oh. Ok.

No exemplo 3, Leonardo sugere que procurem no dicionário como se diz "louco" em inglês, que a dupla não lembra. Diego decide perguntar ao colega Matheus, ao invés de procurar no dicionário, e o colega lhes fornece a resposta. Assim, nesse caso, o colega serviu de fonte externa, auxiliando a resolver uma dúvida da dupla.

Negrito palavra enfatizada na fala.

( ) comentários da pesquisadora. 
Exemplo 3 - Sessão de revisão por pares 2 - 24 de junho - História - Dupla 2

Diego: "Getting" <é com dois "t"s, né>? (parte inaudível da conversa dos dois). "He was getting, more... more"... <como é que é "louco">?

Leonardo: What?

Diego: 〈Louco, louco, louco > (cantarolando). 〈Demorou>.

Leonardo: <Louco >? Dictionary!

Diego: Why dictionary? Matheus! (levanta e vai perguntar para o colega Matheus). <Viu, o Matheus não precisa dicionário>.

Por fim, no exemplo 4, o participante Pedro consulta a professora em relação a uma dúvida no uso da expressão "in other way" utilizada por Nathan. A professora sugere que usem o livro como fonte externa de consulta, para buscar uma expressão mais adequada. Pedro pega seu livro didático, onde encontra outras sugestões que pode dar a Nathan, como vemos a seguir.

Exemplo 4 - Sessão de revisão por pares 5 - 11 de setembro - Redação - Dupla 3

Pedro: I think you have to substitute "in other way the white lies". Don't know (chama a professora).

Professora: Yes?

Pedro: Here, "in other way the white lies are used frequently by everyone".

Professora: Ok. What is your doubt?

Pedro: Is this correct, "in other way"?

Professora: Why don't you use some of the expressions from page 67? Nathan, take a look there. Get your book. There is one expression that means this, that you wanna say, but it is better, because as Pedro said, this is not usual. I understand what you want to say, but the correct expression is on the book.

Pedro (levanta e busca o livro para que ambos olhem). It's the last paragraph (olham no livro).

Pedro: You could put "to sum up" here.

\section{O uso da L1}

Apesar de sua infrequência nas interações, a L1 foi utilizada pelas duplas - em algumas mais, em outras menos - como artefato simbólico para mediar a tarefa. O uso da L1 possibilita que o aprendiz complete a tarefa com mais eficácia e permite que ele mantenha o diálogo e externalize seu pensamento, além de ser uma ferramenta de mediação que irá auxiliar a mediar comportamentos. Ela pode ainda auxiliar a dar andamento à tarefa, a focar a 
atenção, a negociar vocabulário e gramática da L2 e a melhorar as relações interpessoais (SWAIN; LAPKIN, 1998; 2000).

Em alguns exemplos retirados das interações, é possível perceber que a língua portuguesa foi utilizada com esses objetivos. No exemplo 5, Nathan pergunta a Pedro qual o significado da expressão que ele está sugerindo, e Pedro usa a L1 para explicar o significado da expressão desconhecida.

Exemplo 5 - Sessão de revisão por pares 5 - 11 de setembro - Redação - Dupla 3

Pedro: You could put "to sum up" here.

Nathan: Yes, but what's the meaning?

Pedro: <Em resumo. Para resumir> (ficam pensando). You could put "to summarize". I think it is better "to summarize". "In conclusion"...

Também no exemplo 6, a L1 é usada para esclarecer o sentido da palavra. No entanto, no caso a seguir, a pergunta de Fátima, querendo saber o que Jéssica quis dizer, pode não ter sido por não entender o sentido da palavra, mas por não entender seu uso, já que Jéssica usou o adjetivo "alive" com o intuito de usar o substantivo "survival" - o que só é possível perceber por ela ter usado a palavra em português para responder a pergunta de Fátima.

Exemplo 6 - Sessão de revisão por pares 1 - 04 de junho - Resenha - Dupla 4

Fátima: And for the alive, you mean life?

Jéssica: Alive, 〈sobrevivência〉.

Por fim, no exemplo 7, Leonardo usa a L1 para deixar claro para Diego o porquê de ele estar sugerindo que os verbos estivessem no passado. Ao traduzir, ele chama a atenção de seu interlocutor para o fato de que a história deveria ser no passado.

Exemplo 7 - Sessão de revisão por pares 2 - 24 de junho - História - Dupla 2

Leonardo: Here, I put the verbs in the past because the story is in the past... here, "his friends had locked", <eles trancaram>, "locked Bruno on the closet and went out". <Oh, aqui tu usou o verbo>, but here you don't use.

Como foi possível perceber nos exemplos, assim como na análise geral das interações, os participantes foram criteriosos em relação a quando usar a L1, o fizeram com pouca 
frequência e nas situações em que o fizeram, em geral, a L1 foi uma ferramenta útil à negociação de sentido e à colaboração para a revisão dos textos.

\section{A utilização da scaffolding}

Durante a execução da tarefa, no processo de correção, os alunos se ajudavam por meio de estruturas de apoio (scaffolding ou andaimento). $\mathrm{O}$ apoio fornecido um ao outro era mediado pela língua, permitindo a comunicação e coordenando as ações dos participantes da situação de aprendizagem (WELLS, 1999). Os exemplos que serão apresentados a seguir representam o que Donato (1994) chamou de andaimento coletivo, já que neles perceberemos que os aprendizes irão unir seus conhecimentos parciais da L2 para tomar decisões que dizem respeito a vocabulário e estruturas linguísticas do texto de um deles. A construção de conhecimento se dá por meio de diálogo e colaboração, sem a identificação de um membro mais experiente no par (LANTOLF; THORNE, 2006)

$\mathrm{O}$ andaimento pode ser percebido através de diferentes ações por parte dos participantes. São elas: pedir ou dar conselho ou sugestão, responder a conselho ou sugestão, pedir e dar esclarecimento, reafirmar algo falado ou escrito pelo colega e fazer ou responder a perguntas. Passaremos a analisar alguns trechos das interações em que essas estruturas de apoio aparecem. Para fins de análise, não irei separar os exemplos por tipo de apoio, pois perceberemos que em um mesmo trecho, teremos exemplos de mais de um tipo.

No exemplo 8, é possível verificar um participante pedindo sugestão e o outro dando a sugestão solicitada e fazendo uma pergunta.

Exemplo 8 - Sessão de revisão por pares 2 - 24 de junho - História - Dupla 1

Ângelo: Ah, that's ok. Where I can put the direct speech and reported speech in my text? You are the teacher (referindo-se ao par), you have to do this.

Bruno (olhando para o texto de Ângelo): Here... "and then Leo said... asked... asked to Caetano if he was good". Between "sink" and "and".

Ângelo (pensando).

Bruno: Understood?

Ângelo: (discorda).

Bruno: No? You put like... "he throwed up beef in the floor and in the sink. Then Leo asked to Caetano if he was good."

Ângelo: $O k$.

Bruno: And then you put "and who had to clean it was me". Understood? 
Ângelo pede uma sugestão de onde incluir em seu texto discurso direto e indireto, como solicitado pela tarefa. Bruno sugere uma frase em que pode incluir o discurso indireto e duas vezes confirma com Ângelo se entendeu, através da pergunta “understood?".

Nos pedidos de conselho, os autores do texto geralmente pediam ao leitor alguma opinião em relação ao que haviam escrito. Ao dar sugestões, os leitores, por vezes, o faziam ao responder os pedidos de conselho, como no exemplo 8, ou de forma espontânea, ao exercer seu papel de revisor. Além de fazer perguntas para pedir conselhos ou para compreender algo que não estava claro no texto, outras perguntas eram feitas com o objetivo de verificar se o autor do texto concordava com a sugestão, se entendia a sugestão (exemplo 8) ou se compreendia o que estava escrito no texto ou na lista de revisão. Há ainda exemplos em que perguntas foram feitas quando não sabiam dizer algo em inglês.

No exemplo 9, temos novamente uma sugestão fornecida pelo leitor-revisor ao escritor do texto, desta vez, sem que houvesse um pedido de sugestão.

Exemplo 9 - Sessão de revisão por pares 3 - 10 de julho - Email - Dupla 2

Leonardo: Here you put to this paragraph and here and this comma, you put a comma here, don't have this comma.

Diego: $O k$.

Leonardo: Then, here, I don't understand...

Diego: Way, <caminho>.

Além da sugestão, Leonardo faz também um pedido de esclarecimento e Diego fornece esse esclarecimento. Nos pedidos de esclarecimento, o leitor pede ao colega que explique algum aspecto não compreendido do texto ou esclareça alguma palavra não compreendida ou desconhecida, como aconteceu no exemplo 9.

Outra estratégia de apoio é reafirmar algo falado ou escrito pelo colega. No exemplo 10, podemos perceber que Jéssica, atuando como leitora, reafirma o que foi falado por Fátima, para se certificar de que compreendeu e de que esta é a forma mais adequada.

Exemplo 10 - Sessão de revisão por pares 2 - 24 de junho - História - Dupla 4

Fátima: Ahm... I think that I am going to put that the lamp is toxic... "The lamp's powder is toxic"?

Jéssica: "The lamp's powder".

Fátima: Yes, I know. "The lamp's powder".

Jéssica: "The lamp's powder is toxic" or "The lamp is toxic." I don't know if powder is like this. 
Fátima: Yes, I guess.

No exemplo 11, temos novamente um exemplo de reafirmação, seguido por um pedido de esclarecimento. Leonardo repete a palavra "surprised" como forma de indicar que entendeu, pois primeiramente não havia entendido e havia solicitado o esclarecimento. Em seguida, solicita novamente o esclarecimento para outra palavra que não entendeu, que Leonardo informa ser a palavra "was".

Exemplo 11 - Sessão de revisão por pares 2 - 24 de junho - História - Dupla 2

Leonardo: Here I put "when" because "a funny thing happened when..." You put this wrong. Here, this word I don't understand.

Diego: "Surprised".

Leonardo: "Surprised”. And this word? The same of this, but I don't understand.

Diego: (lendo) "Because was"... is "was"

Leonardo: "Was"?

Diego: (consente).

Por fim, temos mais um exemplo de pergunta, em que Diego questiona Leonardo sobre a ortografia da palavra "wrong".

Exemplo 12 - Sessão de revisão por pares 3 - 10 de julho - Email - Dupla 2

Diego: How you can say "wrong”?

Leonardo: What?

Diego: <Não sei como se escreve > "wrong".

Leonardo: "Wrong”? (escreve na folha do colega).

Ao analisar todos esses exemplos em que os participantes se ajudaram por meio de estruturas de apoio, é possível perceber que, ao trabalhar juntos, os participantes parecem ter alcançado níveis mais elevados de desempenho através da assistência mútua, como sugerido por Ohta (2000). Esse conhecimento co-construído pode vir a ser utilizado em atividades subsequentes de forma independente, como sugerido por Donato (1994), e esse seria um grande ganho da atividade colaborativa desenvolvida e um passo dos aprendizes rumo a uma maior autorregulação no uso da L2 e no desenvolvimento da autonomia como aprendiz de L2.

\section{O apoio no conhecimento metalinguístico}


Em alguns exemplos, podemos verificar que os participantes se apoiam no conhecimento metalinguístico como forma de andaimento ao corrigir o texto do colega, justificando sua sugestão ou refutando alguma correção que não lhes parecia válida. Em muitos desses casos, os participantes usavam a língua para refletir sobre seu uso, sem necessariamente usar uma terminologia metalinguística. De acordo com Swain e Lapkin (2002), trata-se do uso de metafala (metatalk), ou seja, a língua sendo usada para refletir de forma consciente sobre seu uso. De acordo com as autoras, é um diálogo colaborativo no qual os participantes estão engajados na resolução de um problema.

No exemplo seguinte, Leonardo está explicando para Diego que ele deveria ter usado a forma "doesn't $t$ " para a frase negativa, a fim de concordar com o sujeito "Bruno". Ele não usa termos como "sujeito", " 3 a pessoa" ou outros, mas explica que a forma "don't" não é a apropriada para a situação e “doesn' $t$ ” sim.

Exemplo 13 - Sessão de revisão por pares 2 - 24 de junho - História - Dupla 2

Leonardo: Here, you put "Bruno do not", is "doesn't". "Do not" is "don't", but "don't" is not appropriate of Bruno, is "doesn't". "Went to the school because his mom was scary to repeat that day" and conclusion?

Diego: Yeah. I don't put.

Já no exemplo 14, Jéssica explica a Fátima o uso inadequado do plural para o substantivo "man", cujo plural é irregular. Ela explica que "man" é a forma do singular e ela deveria ter usado a forma "men".

Exemplo 14 - Sessão de revisão por pares 2 - 24 de junho - História - Dupla 4

Fátima: Jéssica, "mans"?

Jéssica: "Men", with "e".

Fátima: With "s", I put?

Jéssica: No. It's like, "man" with " $a$ " is in the singular and "men" with " $e$ " is...

Fátima (ri de si mesma quando percebe sua falha)

Jéssica (ao comentar com um colega que passa por elas) She wrote "mans", $M-A-N-S$.

\section{O uso da fala privada}

A última estratégia mediadora a ser analisada é o uso da fala privada. Esse uso acontecia quando a intenção do aluno era refletir sobre o que estava lendo ou sobre alguma sugestão que recebeu, sem a intenção de se dirigir ao seu interlocutor. Como os participantes 
permaneciam próximos um ao outro, não só enquanto interagiam sobre as sugestões, mas também durante todo o processo de reescrita, muitas vezes a fala privada, ao ser externalizada por um dos participantes, mesmo sem a intenção de se dirigir ao interlocutor, acabava dando início a uma nova interação em relação às produções.

De acordo com Swain, Kinnear e Steinman (2011), tanto a fala com outros quanto a fala consigo próprio, que as autoras denominam, respectivamente, diálogo colaborativo e fala privada, são formas de lingualizar, sendo que a fala privada, como forma de comunicação intrapessoal, irá auxiliar a mediar os processos de pensamento. Ohta (1995) afirma que a fala privada irá organizar e dirigir essa atividade mental do falante.

No exemplo 15, Leonardo está lendo o texto de Diego em voz alta para si próprio, a fim de verificar a existência de inadequações ou trechos não compreendidos e anotar na lista de revisão para posterior discussão com o colega. Ao fazê-lo, repete a palavra “death" para si próprio com entonação de pergunta. Diego, que nesse momento está a seu lado, percebe que Leonardo está se questionando sobre a palavra "death" e interrompe o pensamento do colega para esclarecer o que quis dizer.

Exemplo 15 - Sessão de revisão por pares 5 - 11 de setembro - Redação - Dupla 2

Leonardo: "Some people think that the honesty is the best way to a good life, but a situation of death"... "death"?

Diego: Yes, “death”, 〈morte>.

No exemplo a seguir, Nathan devolve a lista de revisão e o texto revisado para Pedro, quando têm a seguinte interação:

Exemplo 16 - Sessão de revisão por pares 5 - 11 de setembro - Redação - Dupla 3

Nathan: I wrote this.

Pedro: I know.

Nathan: No that it's wrong, but you have to substitute some "that".

Pedro: Ok. "But nowadays is more normal"... "Honesty can be very useful"... (fica lendo seu texto) Just let me... "special person" (anota algo em seu texto). "In conclusion, on certain moments, it depends on the moment and time"... (assinala algo e relê) "In conclusion, it depends on the moment and time". Ok.

Nathan explica, ao entregar a lista, que essas são suas sugestões (I wrote this). Ele também explica que não acha que esteja errado, mas que o colega deveria substituir a palavra "that" que foi muito usada. Pedro recebe a lista de revisão e a sugestão, respondendo com um 
"ok", e passa a revisar seu próprio texto. Ele vai lendo em voz alta para si próprio o seu texto, a fim de revisá-lo e, ao fazê-lo, acaba por modificar a frase final, excluindo o trecho "on certain moments" e, assim, evitando a repetição da palavra "moments", algo que não foi sugerido por Nathan, mas foi percebido por Diego ao reler seu texto para si próprio em voz alta.

\section{Considerações finais}

A prática de revisão por pares analisada neste estudo foi implementada no contexto pesquisado, conforme mencionado na introdução deste artigo, como uma forma de tentar promover o avanço contínuo do desenvolvimento da proficiência dos aprendizes na L2, ao fornecer mais oportunidade de participação dos alunos em interações que favoreçam o processo de aprendizagem de L2. No entanto, o estudo cujos exemplos são apresentados neste artigo não teve como objetivo medir ou comprovar se houve aprendizagem.

Existem estudos semelhantes que realizam pré e pós-testes em itens linguísticos específicos e comparam resultados de antes e depois da prática de revisão por pares ou escrita colaborativa, a fim de verificar se ocorreu aprendizagem. Em se tratando de aprendizagem de L2 em uma perspectiva sociocultural, na qual a língua serve para agir no mundo e sua aquisição se dá através da interação, penso ser difícil de se atribuir a somente uma estratégia pedagógica o mérito pela ocorrência de aprendizagem em um estudo longitudinal. Enquanto os aprendizes participam por alguns meses de um estudo como este, sua exposição à L2 e suas oportunidades de uso e prática são diversas e não estão limitadas à atividade que está sendo proposta pelo estudo. Dessa forma, a meu ver, não seria possível afirmar através de pré e póstestes que melhores resultados aconteceram em função de uma prática específica em que os aprendizes estavam envolvidos.

Guk e Kellogg (2007) afirmam que, ao falar e escrever, somos capazes de alcançar um entendimento novo e mais aprofundado de um fenômeno complexo e assim, planejar e organizar esses conhecimentos para o futuro com base nessas experiências passadas. De acordo com Foster e Ohta (2005), a assistência fornecida na prática de revisão por pares cria oportunidade de uma performance conjunta que deve ser vista como um importante precursor da produção individual.

Além disso, de acordo com Swain (1998), pode-se dizer que episódios em que os aprendizes refletem conscientemente sobre a língua que estão produzindo são fontes de aprendizagem. Também nesse sentido, Liu e Hansen (2002) afirmam que as pesquisas sobre a 
prática de revisão por pares têm evidenciado que os aprendizes empregam uma variedade de funções linguísticas e negociam sentido durante as atividades, utilizando-se das estratégias mediadoras e isso indicaria que o desenvolvimento linguístico está acontecendo, mesmo se esse não é imediatamente aparente. Por fim, de acordo com Storch (2013), a escrita, ou no caso deste estudo, a reescrita colaborativa não é só uma atividade de produção ou revisão conjunta do texto produzido. No contexto de aprendizagem de L2, a atividade inclui aprendizagem de novo vocabulário, novas maneiras de expressar ideias, bem como a aquisição de uma compreensão mais aprofundada de certas estruturas e convenções gramaticais ou um maior controle sobre o uso dessas estruturas. Assim, poder-se-ia dizer que a prática de revisão por pares proporcionou o uso de estratégias mediadoras indicativas do processo de desenvolvimento linguístico que, combinados com demais dados da pesquisa (BLOS BOLZAN, 2016) da qual esse recorte faz parte, aponta que a prática de revisão por pares proporciona não só a possibilidade de revisar e aperfeiçoar os textos, mas também oportunidades de aprendizagem.

\section{REFERÊNCIAS}

BLOS BOLZAN, D. Colaboração na produção escrita em segunda língua: uma proposta de revisão por pares para a aula de língua inglesa em uma escola com currículo bilíngue. Trabalho de conclusão de curso (Tese de Doutorado). Porto Alegre. PPGLet/UFRGS, 2016. 219p.

CAVALCANTI, M. C. Estudos sobre educação bilíngue e escolarização em contextos de minorias linguísticas no Brasil. D.E.L.T.A., São Paulo, v. 15, p. 387-395, 1999.

DONATO, R. Collective scaffolding in second language learning. In J. P. LANTOLF; APPEL, G. (Eds.). Vygotskian approaches to second language research. Norwood, NJ: Ablex, 1994. p. 33-56

FOSTER, P.; OHTA, A. S. Negotiation for meaning and peer assistance in second language classrooms. Applied linguistics, v. 26, n. 3, p. 402-430, 2005.

GUK, I.; KELLOGG, D. The ZPD and whole class teaching: Teacher-led and student-led interactional mediation of tasks. Language Teaching Research, v. 11, n. 3, p. 281-299, 2007. HORNBERGER, N. H. Extending enrichment bilingual education: Revisiting typologies and redirecting policy. Bilingual education: Essays in honour of Joshua A. Fishman on the occasion of his 65th birthday, v. 1, p. 215-234, 1991.

KOWAL, M.; SWAIN, M. Using collaborative language production tasks to promote students' language awareness. Language Awareness, v. 3, n. 2, p. 73-93, 1994.

LANTOLF, J. P. Introducing sociocultural theory. In: LANTOLF, J. P. (Ed.) Sociocultural Theory and Language Learning. New York: Oxford, 2000. p. 1-25

LANTOLF, J. P.; THORNE, S. L. Sociocultural theory and second language learning. In: VAN PATTEN, B.; WILLIAMS, J. (Eds.) Theories in second language acquisition. Mahwah, NJ: Erlbaum, 2006. p. 201-224

LIU, J; HANSEN, J. Peer response in second language writing classrooms. Ann Arbor: University of Michigan Press, 2002. 
OHTA, A. S. Applying Sociocultural Theory to an Analysis of Learner Discourse: LearnerLearner Collaborative Interaction in the Zone of Proximal Development. Issues in Applied Linguistics, v. 6, n. 2, p. 93-121, 1995.

OHTA, A. S. Rethinking interaction in SLA: Developmentally appropriate assistance in the zone of proximal development and the acquisition of L2 grammar. . In: LANTOLF, J. P. (Ed.) Sociocultural Theory and Language Learning. New York: Oxford, 2000. p. 51-78

SATO, M. Beliefs about peer interaction and peer corrective feedback: efficacy of classroom intervention. The Modern Language Journal. v. 97, n. 3, p. 611-633, 2013.

STORCH, N. Collaborative writing in L2 classrooms. Bristol: Multilingual Matters, 2013.

SWAIN, M. Communicative competence: some roles of comprehensible input and comprehensible output in development. In: GASS, S. MADDEN, C. G. (Eds). Input in Second Language Acquisition. Rowley: Newbury House Publishers, 1985. p. 235-153

SWAIN. M. Collaborative dialogue: its contribution to second language learning. Revista Canaria de Estudios Ingleses. n. 34, p. 115-132, 1997.

SWAIN, M. Focus on form through conscious reflection. Focus on form in classroom second language acquisition, p. 64-81, 1998.

SWAIN, M. The output hypothesis and beyond: Mediating acquisition through collaborative dialogue. In: LANTOLF, J. P. (Ed.) Sociocultural Theory and Language Learning. New York: Oxford, 2000. p. 97-114

SWAIN, M.; LAPKIN, S. Problems in output and the cognitive processes they generate: A step towards second language learning. Applied Linguistics, v. 16, p. 371-391, 1995.

SWAIN, M.; LAPKIN, S. Interaction and second language learning: Two adolescent French immersion students working together. Modern Language Journal, v. 82, n. 3, p. 320-337, 1998.

SWAIN, M.; LAPKIN, S. Task-based second language learning: The uses of the first language. Language Teaching Research, v. 4, p. 251-274, 2000.

SWAIN, M.; LAPKIN, S. Talking it through: two French immersion learners' response to reformulation. International Journal of Educational Research, v. 37, p. 285-304, 2002. SWAIN, M.; KINNEAR, P.; STEINMAN, L. Sociocultural theory in second language education: An introduction through narratives. Bristol, England: Multilingual Matters, 2011. VYGOTSKY, L. S. Mind in society: The development of higher psychological processes. Cambridge, MA. Harvard University Press, 1978.

WELLS, G. Dialogic inquiry: Towards a socio-cultural practice and theory of education. Cambridge: Cambridge University Press, 1999. 